\title{
Variable and Constant Features on Titan from HST
}

Ralph D. Lorenz, Mark T. Lemmon, Peter H. Smith

Lunar and Planetary Laboratory, University of Arizona, Tucson, AZ 85721, USA

\begin{abstract}
Observations with HST WFPC-2 from 1994 to 1997 show a number of features which can be attributed to the surface (notably the large as-yet-unnamed bright region, several smaller bright areas and a number of dark regions). The delineation of these features in several datasets is presented. Other features are variable and are due to atmospheric phenomena: these include the total brightness of Titan (drop by $5 \% 1994-1997$ at $350 \mathrm{~nm}$; increase by $7 \%$ at $889 \mathrm{~nm}$ ), the hemispheric north-south asymmetry, and the hint of a south polar hood.

Additionally, small variable features, apparently due to clouds, are documented. As well as variability from one year to the next, these show anomalous center-to-limb brightness behaviour, and a $673 / 940 \mathrm{~nm}$ color much bluer than the large surface feature, consistent with clouds in the troposphere.
\end{abstract}

